\title{
Disminución de la agresividad en estudiantes de primaria: El programa "Fortaleciéndome"
}

\section{Decrease in aggressiveness in elementary students: The "Strengthening" program}

Eddy Eugenio García García

Universidad César Vallejo, Trujillo, Perú

ORCID: https://orcid.org/0000-0003-3267-6980

Alejandro Cruzata-Martínez*

Universidad San Ignacio de Loyola, Lima, Perú

ORCID: https://orcid.org/0000-0003-0104-0496

Roberto Santiago Bellido García

Universidad San Ignacio de Loyola, Lima, Perú

ORCID: https://orcid.org/0000-0002-1417-3477

Luis Gerardo Rejas Borjas

Universidad Nacional Mayor de San Marcos, Lima, Perú

ORCID: https://orcid.org/0000-0002-7679-4973

Recibido 01-03-20 Revisado 12-04-20 Aprobado 14-06-20 En línea 03-07-20

*Correspondencia

Email: alejandrocruzatamartinez@yahoo.es
Citar como:

García, E., Cruzata-Martínez., A., Bellido, R., \& Rejas, L. (2020). Disminución de la agresividad en estudiantes de primaria: El programa "Fortaleciéndome" Propósitos y

Representaciones, $8(2), \quad$ e559.

http://dx.doi.org/10.20511/pyr2020.v8n2.559 


\section{Resumen}

El objetivo fue determinar la influencia significativa del programa "Fortaleciéndome" en el nivel de agresividad. La muestra estuvo conformada por estudiantes de una escuela de Comas, con edades entre los 10 y 11 años. Se empleó como instrumento el Cuestionario de Agresividad de Buss y Perry (1992, adaptación a la realidad peruana de Matalinares et al., 2012). Se planificó un diseño cuasiexperimental, en el pretest los resultados fueron similares tanto en el grupo control como en el grupo experimental (niveles promedio y alto, respectivamente). En el postest los resultados fueron distintos: el grupo control mantuvo su nivel inicial, pero el grupo experimental redujo su nivel de agresividad. Los resultados estadísticos obtenidos $(\mathrm{Z}=5,957>1,6706$ y $\mathrm{p}=$ $0.000<0.05)$ permiten afirmar que la aplicación del programa "Fortaleciéndome" sí tuvo una influencia significativa en la disminución de la agresividad, y en sus dimensiones agresividad física, agresividad verbal, ira y hostilidad.

Palabras clave: Agresividad; Habilidades sociales; Inadaptación; Escuela primaria.

\section{Summary}

The objective was to determine the significant influence of the "Strengthening Me" program on the level of aggressiveness. The sample consisted of students from a school of Comas, aged between 10 and 11 years. The Buss and Perry Aggression Questionnaire (1992, adaptation to the Peruvian reality of Matalinares et al, 2002) was used as an instrument. A quasi-experimental design was planned, in the pretest the results were similar both in the control group and in the experimental group (average and high levels, respectively). In the posttest the results were different: the control group maintained its initial level, but the experimental group reduced its level of aggressiveness. The statistical results obtained $(Z=5.957>1.6706$ and $p=0.000<0.05)$ allow us to affirm that the application of the "Strengthening Me" program did have a significant influence on the decrease in aggressiveness, and in its dimensions physical aggressiveness, verbal aggressiveness, anger and hostility.

Keywords: Aggressiveness; Social Skills; Maladjustment; Primary Schools.

\section{Introducción}

Según la teoría sociológica, la agresión es interpretada como un producto de los acontecimientos culturales, políticos y económicos de la sociedad (Dollar, Doob, Miller, Mowrer \& Sears, 1939). Añádase la alta influencia de los medios audiovisuales, está demostrado que la exposición continuada a la violencia audiovisual incrementa la agresividad interpersonal. Varios estudios (López \& Sabater, 2014; Stein, Friedrich \& Vondracek, 1972) apoyan esta idea. Nada más vigente para nuestro tiempo, pues somos testigos de expresiones agresivas en todo ámbito social, sea en lo personal, familiar, laboral; en toda latitud continental trascendiendo condiciones económicas, de género, de creencias, de clases sociales, entre otras.

De manera particular en el ámbito educativo, sabemos que la violencia escolar es un problema global, puesto que cada año 246 millones de niños y jóvenes experimentan violencia escolar (Organización de las Naciones Unidas, 2017). En América Latina, el 51,12\% de los estudiantes de sexto grado de primaria fueron víctimas de algún maltrato, siendo Argentina la que presenta la frecuencia más alta, la cual alcanza el 58,62\% (Román \& Murillo, 2011). En el Perú, durante setiembre del 2013 y diciembre del 2018 se reportaron 26285 casos de violencia escolar. El número total de casos reportados entre enero y diciembre del 2017 fue de 5591. Para el año 2018, la cifra superó los 9512 casos, mientras que hasta julio del 2019 ya tenemos 4931 casos, es decir 27 niños al día sufren agresividad en las escuelas (Ministerio de Educación, 2019). Sin duda, las cifras son alarmantes, como también lo son el descuido del Estado y la pasividad con que la población contempla este problema. A pesar de los esfuerzos, no existen políticas de Estado serias 
que ataquen el problema de fondo, enraizado en la discriminación económica y social hacia la población, lo que no permite una convivencia realmente democrática a partir de la formación de un ciudadano verdaderamente moral.

Etimológicamente, la palabra agresividad se deriva del latín aggredior, que significa "ir o acometer contra otro". El diccionario de la Real Academia Española (2019) definió el término agresivo como el comportamiento humano "propenso a faltar al respeto, a ofender o a provocar a los demás". Es decir, la voluntad de una persona por imponerse a otra u otras, al margen que si ello signifique causar o generar daño ya sea físico o psicológico.

Por otro lado, la agresividad también es definida como un comportamiento individual que tiene el propósito de dañar a otros por su forma física, verbal y en daños a la propiedad (Atkinson, Atkinson \& Hilgard, 1983; Fadila, 2013; Kartono, 2005; Novita, 2017). Hoy sabemos que la conducta agresiva también puede ser premeditada y organizada con la participación de varios individuos y el daño no solo se dirige a los demás, sino también a sí mismos. Quien se comporta agresivamente tiene poca tolerancia a la frustración y tiende a reaccionar rápidamente con un impulso de agresión, y es menos capaz de hacer auto reflexión (Currie, 2004). La comprensión del problema necesita de una perspectiva social, por lo que la Organización Panamericana de la Salud (2016) sostuvo que la agresividad es un problema complejo, en el que existen múltiples factores implicados, entre ellos los antecedentes de comportamientos agresivos o de haber sufrido maltrato, lo que estaría relacionado con el comportamiento violento en la adultez; de hecho contiene elementos multidimensionales y multicausales para su aparición, desarrollo, expresión y control. La agresividad no sólo se manifiesta con golpes, robos, insultos; también son conductas agresivas la discriminación, las burlas, los apodos, el ignorar al otro y a la otra, el aplicar la "ley del hielo" (Díaz, 2012. p.2).

Otro aspecto que nos da amplitud sobre el concepto de agresividad tiene que ver con el factor cultural o contextual, por ello en ciertas sociedades lo que consideraríamos comportamiento agresivo podría ser legítima defensa, una costumbre cultural o un ritual religioso (Ledesma, 1980). A pesar de esta relatividad, toda conducta con la que se pretenda infligir un daño es agresiva. Por tanto, al tratar el concepto agresividad también debemos considerar el desarrollo histórico de la persona y del contexto en el que se desenvuelve, es decir, el desarrollo filogenético y ontogenético de su personalidad.

Hablar de agresividad implica identificar a sus actores y el rol que cumple cada uno de ellos. Los agresores han sido caracterizados por su posición de liderazgo y poder, el poco control de la agresión y la necesidad de dominio que ejercen hacia los otros (Cerezo, 2009; Leff, Kupersmidt, Patterson \& Power, 1999 en Pérez, 2011). Quiere decir que el agresor normalmente no actúa solo, sino en compañía activa o pasiva de otros frente a su víctima, lidera un grupo, se presenta como el más fuerte, y establece el sistema de valores de quienes lo admiran y festejan lo que hace (Oliveros \& Barrientos, 2007). Por otro lado, el actor "complementario" es la víctima, la cual se caracteriza por presentar sumisión y pasividad ante su agresor, es decir, por mostrar retraimiento ante la situación de violencia (Schwarts, Proctor \& Chien, 2001 en Salgado, 2012, p.137). Finalmente, tenemos a los espectadores quienes presentan una conducta pasiva o que oculta el problema, quienes, fieles a una negativa "ley del silencio", favorecen y refuerzan la agresión y el conflicto (Prieto, Carrillo \& Jiménez, 2005, p. 1033); tal vez "la consecuencia principal entre los observadores es el miedo a ser víctimas" (Sáenz, 2010, p.10). Así, el agresor se fortalece a partir del temor de los otros, se posiciona socialmente desde la violencia y se daña a sí mismo como persona a partir del daño que va generando en los demás. Podríamos decir entonces que el agresor también es, al mismo tiempo, "víctima", en tanto degeneración de su personalidad; cumpliendo así, un doble rol.

La agresividad escolar es un reflejo de la descomposición de la sociedad, en el marco de un Estado de derecho débil, una estructura socioeconómica incapaz de satisfacer las necesidades básicas de la población y una ruptura del tejido social que desencadena la lucha por espacios 
alternos de sobrevivencia y reorganización sociopolítica (Tello, 2005). Entendida así, la agresividad escolar es el reflejo de una crisis mayor que se acrecienta agudizando el problema de las relaciones de convivencia a todo nivel, incluida la escuela. La conducta agresiva que muestran algunos adolescentes hacia sus iguales en el contexto escolar hace referencia a un conjunto de comportamientos dirigido hacia otros compañeros, con el objeto de causar un daño intencionado a nivel físico, verbal o relacional (Estévez, Jiménez \& Musitu, 2008; Little, Henrich, Jones \& Hawley, 2003). Por tanto, una sociedad en descomposición implica un ciudadano orientado también hacia esa condición, más aún en grupos vulnerables como los preadolescentes, susceptibles de ser influenciados por modelos alienantes que perturban su personalidad y sus actitudes con los demás.

Se ha considerado que la agresión entre compañeros es la forma más común de violencia escolar (Nansel et al., 2001). A su vez, es la que presenta mayor diversidad de formas y la que se presenta con mayor frecuencia, considerando que no todas son explícitas pues existen grados altos de agresión oculta, no denunciada o invisibilizada principalmente por temor a represalias y revictimización. Esta situación genera también consecuencias al convertirse en un obstáculo importante para el desarrollo del proceso de enseñanza-aprendizaje y el ajuste psicosocial y escolar, tanto de las víctimas como de los agresores (Astor, Pitner, Benbenishty, \& Meyer, 2002). No por puro gusto, Freire (1997) señalaba que "si se respeta la naturaleza del ser humano, la enseñanza de los contenidos no puede darse alejada de la formación moral de los educandos" (p. 34). Es decir, la escuela y la sociedad deben priorizar la promoción de una convivencia sana y democrática entre los escolares, donde el diálogo y la participación protagónica sea un medio para construir relaciones donde prime el respeto y la tolerancia; es decir la formación moral que -a fin de cuentas- debe ser la que vaya por delante de cualquier otra dimensión de la formación de la personalidad de todo ser humano.

Esta situación genera la necesidad de promover la puesta en práctica de programas de intervención que fomenten la buena convivencia escolar, pues las prácticas agresivas entre escolares trascienden hacia toda la comunidad educativa. Al respecto, Benítez y Justicia (2006) afirmaron que "el maltrato entre iguales es un problema en los centros educativos cuyas consecuencias afectan a todos los agentes implicados, e indirectamente, al resto de la comunidad educativa que ha de convivir con los efectos derivados del mismo" (p.165). Así, la agresión entre estudiantes tiene impacto institucional y debe ser abordada también en esa misma dimensión, es decir, con la participación de toda la comunidad educativa; entiéndase los docentes, los directivos, las familias y los mismos estudiantes. De no ser así, las consecuencias se agravan y pueden ser mayores a futuro, pues la implicación en conductas agresivas en la escuela se relaciona con posteriores problemas de salud, y con distintos diagnósticos como cuadros de ansiedad y personalidad antisocial. Otros estudios también informan que participar en actos de agresión en la escuela incrementa las tasas de distrés psicológico, depresión, absentismo escolar y actitudes negativas hacia la escuela (Brunstein et al., 2011; Zimmer \& Pronk, 2012).

La agresividad ha sido motivo de análisis desde diversas teorías. Se tiene a las teorías biologicista, social, y de factores situacionales. La teoría biologicista, con Lorenz $(1966,1974)$, sugirió que la agresión surge principalmente de un instinto de lucha heredado, que los seres humanos comparten con otras especies. Es decir, en la base evolutiva, a los animales y al hombre les es inherente el comportamiento agresivo, estando predispuestos a demostrarlo ante la necesidad o la influencia del medio, puesto que requieren conservar su especie y adaptarse a las más diversas circunstancias. Las investigaciones biologicistas suponen que el origen de la agresividad y la conducta del hombre en general están condicionados por el nivel de maduración y su desarrollo, que de ello depende el origen y el grado de agresión que vaya mostrando la persona, mientras que la influencia del medio no es fundamental, aunque puede servir solo como factor secundario.

Con lo anterior, se incluyen factores bioquímicos y genéticos, que en los últimos tiempos han cobrado relevancia. Álvarez (2002) afirmó que "el comportamiento agresivo se desencadena como consecuencia de una serie de procesos bioquímicos que tienen lugar en el interior del 
organismo y en los que desempeñan un papel decisivo las hormonas. Se ha demostrado que la noradrenalina es un agente causal de la agresión". Estas nuevas tendencias surgen para reforzar la concepción biologicista, e insisten en el reduccionismo, por lo que no son válidas para explicar objetivamente el tema tratado. Baron y Byrne (2005), refirieron que, las personas tienen muy diversas maneras de expresar conductas agresivas, como ignorar a los demás, la agresión física, dañar a los otros, etc., razón por la cual afirman que la agresividad no tiene un origen biológico. De ser así, o sea, de tener un componente genético programado predispuesto para la agresividad, las conductas agresivas serían similares y no tan diversas como realmente lo son. A partir de ello, se considera que el ser humano y sus conductas son regulados por la sociedad y la cultura. De tal manera que se propicie las relaciones humanas basados en la convivencia armoniosa y no en el poder de la fuerza (Castrillón \& Vieco, 2002).

La teoría social de la agresividad asume que ésta, además de estar fundamentada en los instintos, en los impulsos y en los estados internos de la persona; también estaría generada por los aspectos externos contextuales y/o sociales, los cuales ejercen influencia en el comportamiento. Las teorías ambientales o sociales entienden que el origen de la agresión se encuentra en el ambiente que rodea al estudiante, es decir, las causas de la agresividad son exógenas. Estas teorías presentan la conducta de agresión como una reacción de emergencia por parte de los alumnos frente a diversos sucesos ambientales o sociales adversos (Berkowitz, 1993).

Según el modelo de la "imitación social" propuesto por Bandura (1973), el comportamiento agresivo es el resultado de un aprendizaje por observación e imitación de un modelo cercano al estudiante. Este va a imitar una conducta agresiva si obtiene beneficios por ella, es decir, si es recompensado positivamente, pero si por el contrario es castigado, disminuirá la probabilidad de agredir a otros. Esta concepción asume un aprendizaje aislado, fuera de contexto, por lo que no toma en cuenta diversos factores que reflejan el carácter multidimensional de la agresividad, es decir, afrontar un problema social y complejo como la agresividad no puede agotarse solo en la imitación.

Baron y Byrne (2005) afirmaron que, el aprendizaje de la agresividad puede proceder de la imitación de modelos agresivos de la televisión, programas con alto contenido violento, además de los modelos parentales. Sin embargo, sostienen que este aprendizaje se adquiere principalmente por la observación de las consecuencias de tales comportamientos, lo cual es asimilado en la experiencia personal. Por tanto, serán la educación y los procesos de intervención culturales los que contribuyan en la formación de la moral de la persona, permitiéndole adquirir habilidades sociales para nuevas formas de comportamiento e interrelación con los demás.

La teoría de los factores situacionales considera que la agresión se ve fuertemente influida por los factores sociales y las características personales, pero también se ve afectada por factores relacionados con la situación o el contexto donde ésta ocurre, incluyendo el grado en que la agresión se considera aceptable en una cultura dada (Cohen, Nisbett, Bowdle \& Schwarz, 1996). Este es un enfoque muy tomado en cuenta, pues considera por ejemplo al ambiente familiar y su historia como factores claves para saber el origen de la agresividad en la persona, no siendo determinante. Los factores situacionales nos permiten contextualizar de mejor manera explicaciones acerca del origen, las posibles causas, el grado de agresividad, las formas de expresión y las consecuencias del mismo. Otros factores asociados al origen y expresión de la agresividad pueden ser el alcoholismo, las drogas, historial de enfermedades, ambiente social, objetivos y metas logradas, entre otras. Los estudios de Baron y Byrne (2005) coinciden en que hay diversas causas para explicar la agresividad en las personas, siendo las principales y determinantes, las de índole social, personal y situacional.

El presente trabajo asume que la agresividad se presenta de forma física, verbal, al igual que en forma de ira y hostilidad tanto en contextos sociales como escolares. Estas son, entonces, las dimensiones de la variable agresividad. Según Castillo, Cruz, Peña y Cujiño (2012), "las formas de violencia que se producen en la escuela son tanto físicas como psicológicas y 
generalmente se dan al mismo tiempo" (p. 21). Por tanto, la agresividad física es cualquier comportamiento que tiene como fin herir o humillar a una persona. Es un comportamiento que el individuo ejecuta con el propósito de dañar material o psicológicamente. Ramírez y Andreu (2003) coinciden con esta idea y la catalogan como una agresión de tipo manifiesta.

La agresión verbal pretende causar dolor psicológico, como la humillación, vergüenza y otros sentimientos negativos sobre el yo, que a veces resulta en ataque físico (Piko \& Keresztes, 2006). Los estudios revelan que la manifestación de la agresión verbal más común entre el estudiantado es: los insultos a las condiciones corporales y físicas, asignación de motes/sobrenombres, hablar mal del compañero y amenazas (Gómez, 2005). Así mismo, se puede afirmar que este tipo de agresividad es el que más se puede evidenciar en la cotidianidad y a la vez es el que menos se tiene en cuenta, cuando se plantean alternativas de solución.

La ira es una reacción de irritación, furia o cólera que puede verse incitada por la indignación y el enojo al sentir vulnerados nuestros derechos (Fernández, 1998). El enojo o ira es una emoción humana completamente normal y se puede decir que hasta saludable, mientras no supere ciertos límites; de lo contrario se vuelve destructiva, originando muchos problemas especialmente en las relaciones con otras personas y en general, afectando la calidad de vida. Por ello, la ira sería una respuesta emocional a amenazas reales o imaginarias o una provocación. Puede tener orígenes externos o internos, las circunstancias y experiencias que se presentan (externos), como también los pensamientos, preocupaciones, recuerdos de hechos traumáticos o frustrantes (internos) facilitan la aparición de la ira.

La hostilidad es definida por Coccaro (2003) como los sentimientos que canalizan la emoción de la ira hacia uno o varios objetos, sentimientos que suelen ser bastante duraderos y, especialmente intensos. Coccaro (2003) y Rendón (2008) indican que esta emoción negativa tiene raíz en el rechazo y la provocación de sus pares, y normalmente aparece a falta de estrategias de regulación de las emociones y se vuelve un riesgo poderoso para la agresión. Es así que, la hostilidad supone un comportamiento abusivo y agresivo, que puede transformarse en violencia física o verbal, incluso sin tener motivo aparente y con el deseo de hacer daño.

Plutchik (1980) consideró a la hostilidad como una actitud que mezcla la ira y disgusto, y se ve acompañada de sentimientos tales como indignación, desprecio y resentimiento hacia los demás. Por tanto, la hostilidad o comportamiento hostil, se puede manifestar de varias formas, a veces, actitudes sutiles como frases irónicas o de burla, a veces en forma más directa, es decir, con acciones con el propósito de intimidar o hacer sentir mal a la otra persona. Si la ira y la hostilidad se refieren a sentimientos y actitudes, la agresión implica un paso más allá, puesto que conlleva la aparición de comportamientos destructivos, lesivos o punitivos dirigidos a otras personas u objetos (Miguel, Casado, Cano \& Spielberger, 1997). De esta manera, existe una clara interrelación entre las dimensiones citadas, las cuales son expresión de agresividad, aunque se reconoce que de diferente intensidad. Al respecto, Jenaabadi y Nastiezaie (2011) resumen su naturaleza de la siguiente manera: los ataques verbales y físicos incluyen aspectos conductuales, la ira representa un componente emocional o afectivo, siendo la hostilidad la dimensión cognitiva de la agresión.

\section{Método}

\section{Participantes}

La muestra estuvo formada por 57 estudiantes del $5^{\circ}$ grado de primaria de la institución educativa pública Nuestra Señora de Fátima, cuyas edades fluctúan entre los 10 y 11 años de edad. Comas, al norte de Lima Metropolitana, es el cuarto distrito más poblado con 544000 habitantes (Fondo de las Naciones Unidas para la Infancia, 2019), además de ser el segundo más poblado de Lima Norte. A nivel local, en Comas, 13 de cada 100 niños trabajan, por lo que habría que tener en cuenta que privar a un niño de jugar para forzarlo a trabajar desde edad temprana contribuye a 
producir las condiciones favorables para el resentimiento, la frustración, la carencia de afecto, y un bajo nivel de autoestima personal (Universidad Católica Sedes Sapientiae, 2010). Si a esto se le suma el 16\% de pobreza de Comas entonces el deterioro de los niveles de condiciones de vida de la infancia se convierte en una realidad indiscutible. A nivel nacional, según la Encuesta Nacional de Relaciones Sociales (ENARES), el 75\% de los niños y niñas manifestó haber sido víctimas de violencia física o psicológica por parte de sus otros estudiantes de su colegio (Instituto Nacional de Estadística e Informática, 2015).

La institución educativa pública 2033 Nuestra Señora de Fátima tiene una población estudiantil que, en su mayoría, es de condición socioeconómica media y baja. En ella, la Dirección del centro educativo gestiona un registro de incidencias, este contiene las quejas de los estudiantes respecto al uso de sobrenombres, insultos, golpes, pérdida de objetos personales. El Departamento Psicológico identificó que la mayoría de los niños implicados en estos incidentes provienen de hogares disfuncionales, caracterizados por el abandono familiar, moral, por lo que las conductas mostradas afectan tanto al agresor como al agredido, por ejemplo: inasistencias continuas, bajo rendimiento académico, serias dificultades para la convivencia escolar.

\section{Instrumento}

"Fortaleciéndome" es una adaptación del programa "Capacitación para el Fortalecimiento de Competencias de Jóvenes para el Empleo" (2013), elaborado por el Ministerio de Trabajo y Promoción del Empleo y la Universidad ESAN. El programa en mención cuenta con tres módulos: habilidades cognitivas, habilidades para la construcción, y habilidades socioemocionales, este último es el aspecto medular de "Fortaleciéndome". Esta propuesta contempla técnicas participativas, y tiene como propósito disminuir el nivel de agresividad y mejorar las relaciones interpersonales en niños de 10 u 11 años de edad aproximadamente, en escuelas de la Educación Básica Regular.

Hay una serie de programas encaminados a la prevención de esta problemática desde hace décadas (Cerezo \& Esteban, 1992; Mora \& Ortega, 1997; Pareja, 2002; Viera, Fernández, \& Quevedo, 1989). "Fortaleciéndome" se inserta en ese interés académico, al buscar que los estudiantes adquieran y dominen habilidades sociales que les permitan afrontar cualquier situación que se les presente sin problemas y de manera asertiva. Se busca promover el desarrollo de un equilibrio emocional en los estudiantes cuando interactúen en su ambiente, ya sea familiar, social o escolar a través de la metacognición, generando conductas que favorezcan una convivencia armoniosa, eliminando las conductas agresivas.

\section{Procedimiento}

Se planificó un diseño cuasiexperimental, estableciéndose un grupo experimental y un grupo control, en el que se toman medidas pretest y postest. De acuerdo con García (2001), en las últimas décadas los diseños cuasiexperimentales son cada vez más empleados en la investigación aplicada, sobre todo por su utilidad para la evaluación de la efectividad y eficacia y el mejoramiento de la planificación y el control de los programas educativos.

\section{Descripción de la intervención}

El programa "Fortaleciéndome" está diseñado para ser aplicado durante 20 sesiones, en un tiempo aproximado de 3 meses. El programa consta de tres fases:

A. Primera fase (sensibilización), la cual estará dirigida a los padres, docentes y estudiantes. Con los padres y docentes se realizará un taller sobre la agresividad, donde se les explicará el objetivo del programa y su importancia; asimismo, se buscará que se involucren y que participen a lo largo del programa. Con los estudiantes, se incidirá mucho en la 
motivación, haciendo que afloren sus conocimientos previos, y animándolos para que los pongan en práctica y se les inducirá hacia la práctica de conductas no violentas.

B. Segunda fase (aplicación) en la que se ejecutará lo planificado para las sesiones del programa, dirigidas a los estudiantes del $5^{\circ}$ grado de primaria de la institución educativa pública 2033 Nuestra Señora de Fátima. Cada una de las sesiones contempla el uso de técnicas, dinámicas y estrategias pedagógicas.

C. Tercera fase (evaluación) en la que se podrá verificar los resultados del programa, y analizar su efectividad o su influencia significativa en la reducción del nivel de agresividad de los niños que formaron parte de la muestra. Con los estudiantes, la autoevaluación tendrá como herramienta principal a la reflexión metacognitiva.

Módulo 1. Autoconcepto y autoestima en el programa Fortaleciéndome.

\begin{tabular}{lll}
\hline Nombre de la unidad & Dimensión priorizada & \multicolumn{1}{c}{$\begin{array}{c}\text { Nombre de sesiones } \\
\text { y actividades }\end{array}$} \\
\hline & Autoconocimiento & "Yo soy único y especial" \\
AUTOCONCEPTO & Autoconcepto & "El Bosque" \\
Y AUTOESTIMA & Autoconcepto & "El Reflejo de lo que Pienso" \\
& Autoaceptación & "Aceptándome" \\
& Autoestima & "Carta a mi mejor amigo" \\
\hline
\end{tabular}

Módulo 2. Empatía y asertividad en el programa Fortaleciéndome.

\begin{tabular}{ccc}
\hline Nombre de la unidad & $\begin{array}{c}\text { Dimensión } \\
\text { priorizada }\end{array}$ & $\begin{array}{c}\text { Nombre de sesiones } \\
\text { y actividades }\end{array}$ \\
\hline & Empatía & "Poniéndome en el lugar del otro" \\
EmPATÍA Y & Empatía & "Escuchando atentamente" \\
Asertividad & "Respondiendo Empáticamente" \\
ASERTIVIDAD & $\begin{array}{c}\text { Asertividad } \\
\text { Asertividad }\end{array}$ & "Comunicándome con respeto" \\
&
\end{tabular}

Módulo 3. Inteligencia emocional en el programa Fortaleciéndome.

\begin{tabular}{|c|c|c|}
\hline Nombre de la unidad & Dimensión priorizada & $\begin{array}{c}\text { Nombre de sesiones } \\
\text { y actividades }\end{array}$ \\
\hline \multirow{5}{*}{$\begin{array}{l}\text { INTELIGENCIA } \\
\text { EMOCIONAL }\end{array}$} & Autoconciencia emocional & "Las emociones en mí" \\
\hline & Autoconciencia emocional & "Diccionario emocional" \\
\hline & Control de Impulsos & "Dar vuelta al pensamiento" \\
\hline & Control de Impulsos & "El semáforo" \\
\hline & Manejo del estrés & $\begin{array}{ll}\text { "¿Cuáles } & \text { son } \\
\text { estresores", } & \\
\end{array}$ \\
\hline
\end{tabular}

Módulo 4. Resolución de problemas en el programa Fortaleciéndome.

\begin{tabular}{|c|c|c|c|}
\hline Nombre de la unidad & $\begin{array}{c}\text { Dimensión } \\
\text { priorizada }\end{array}$ & & Nombre de sesiones y actividades \\
\hline \multirow{5}{*}{$\begin{array}{l}\text { RESOLUCIÓN DE } \\
\text { PROBLEMAS }\end{array}$} & Contexto & & "Es problema ¿Sí o No? \\
\hline & Contexto & & "La Isla" \\
\hline & Confianza & & "Tengo - quiero" \\
\hline & Confianza & & "Yo sí puedo" \\
\hline & $\begin{array}{l}\text { Solución } \\
\text { problemas }\end{array}$ & de & "Todo tiene solución" \\
\hline
\end{tabular}




\section{Resultados}

En el presente trabajo, se busca conocer si el programa "Fortaleciéndome" contribuye a disminuir la agresividad, en los estudiantes del $5^{\circ}$ grado de primaria de la institución educativa pública 2033 Nuestra Señora de Fátima. Se estableció un grupo experimental y un grupo control. En ese sentido, un estadístico que evalúa dicha magnitud es el tamaño del efecto (Kirk, 1996). Dada la información en la tabla 1 en el pretest, el cálculo de la probabilidad de superioridad como tamaño del efecto para la U de Mann-Whitney. Este coeficiente indica que la probabilidad que un puntaje seleccionado aleatoriamente de un grupo Y, es superior al seleccionado de un grupo X (ErcegHurn \& Mirosevic, 2008). Su expresión matemática es la siguiente:

$$
P S_{e s t}=\frac{U}{m n}
$$

Dónde: U es la Mann-Whitney; $m$ y n son el número de participantes para cada grupo. En ese sentido, en la tabla 1, que se refiere al pretest para el grupo experimental y grupo control, se aprecian los valores de la probabilidad de superioridad para la variable y sus dimensiones; observándose que la hostilidad presenta el valor más elevado (PSest $=0,47$ ); seguido de la ira (PSest=0,43), agresión física (PSest=0,41); y el más bajo agresión verbal (PSest=0,36). Además, el valor total de agresividad total $(\mathrm{PSest}=0,47)$ es pequeño (Grissom, 1994). Asimismo, en la tabla 1, el p-valor en la variable total y sus dimensiones refieren que no existen diferencias significativas; cuando en realidad está indicando, que existe mucho error para rechazar la afirmación que los rangos son idénticos (hipótesis nula). Mientras que el PSest sugiere que el grupo experimental es superior al control en cada una de las dimensiones; el valor de la variable agresividad total es 0,48, este valor se aproxima a un pequeño efecto (Grissom, 1994). Si se utiliza las normas interpretativas de: No efecto (PSest $\leq=0,0$ ); pequeño (PSest $\geq 0,56$ ); mediano (PSest $\geq$ $0,64)$ y grande (PSest $\geq 0,71)$.

Tabla 1.

Resultados de U de Mann Whitney para el pretest de la escala agresividad total y sus dimensiones con su respectivo tamaño del efecto

\begin{tabular}{|c|c|c|c|c|c|c|}
\hline $\begin{array}{c}\text { Variable y } \\
\text { dimensiones }\end{array}$ & Muestras & Rangos & $\mathrm{n}$ & $\begin{array}{l}\text { U de Mann- } \\
\text { Whitney }\end{array}$ & P-valor & PSest \\
\hline \multirow{2}{*}{$\begin{array}{l}\text { Agresividad } \\
\text { (escala total) }\end{array}$} & Grupo control & 29.47 & 29 & \multirow{2}{*}{392.500} & \multirow{2}{*}{0.829} & \multirow{2}{*}{0.48} \\
\hline & Grupo experimental & 28.52 & 28 & & & \\
\hline \multirow{2}{*}{ Agresividad verbal } & Grupo control & 29.41 & 29 & \multirow{2}{*}{294.500} & \multirow{2}{*}{0.854} & \multirow{2}{*}{0.36} \\
\hline & Grupo experimental & 28.6 & 28 & & & \\
\hline \multirow{2}{*}{ Agresividad física } & Grupo control & 26.29 & 29 & \multirow{2}{*}{330.000} & \multirow{2}{*}{0.223} & \multirow{2}{*}{0.41} \\
\hline & Grupo experimental & 31.62 & 28 & & & \\
\hline \multirow{2}{*}{ Ira } & Grupo control & 31.16 & 29 & \multirow{2}{*}{345.500} & \multirow{2}{*}{0.332} & \multirow{2}{*}{0.43} \\
\hline & Grupo experimental & 26.91 & 28 & & & \\
\hline \multirow{2}{*}{ Hostilidad } & Grupo control & 28.05 & 29 & \multirow{2}{*}{379.500} & \multirow{2}{*}{0.671} & \multirow{2}{*}{0.47} \\
\hline & Grupo experimental & 29.91 & 28 & & & \\
\hline
\end{tabular}

Fuente: Elaboración propia

Nota: $\mathrm{n}=$ tamaño muestral; PSest= Probabilidad de Superioridad (tamaño del efecto).

En la tabla 2, en el postest, para el grupo experimental y grupo control, se aprecian los valores de la probabilidad de superioridad para la variable y sus dimensiones; observándose que 
la agresividad verbal es el valor más elevado (PSest= 0,22); seguido de agresión física (PSest=0,13), ira (PSest=0,08); y el más bajo hostilidad (PSest=0,06). El valor de la variable agresividad total es 0,04, este valor se aproxima a no hay efecto (Grissom, 1994).

Tabla 2.

Resultados de U de Mann Whitney para el postest de la escala agresividad total y sus dimensiones con su respectivo tamaño del efecto

\begin{tabular}{|c|c|c|c|c|c|c|}
\hline $\begin{array}{l}\text { Variable y } \\
\text { dimensiones }\end{array}$ & Muestras & Rangos & $\mathrm{n}$ & $\begin{array}{l}\text { U de Mann- } \\
\text { Whitney }\end{array}$ & P-valor & PSest \\
\hline \multirow{2}{*}{$\begin{array}{l}\text { Agresividad } \\
\text { (escala total) }\end{array}$} & Grupo control & 41.84 & 29 & \multirow{2}{*}{33.500} & \multirow{2}{*}{$0.000 *$} & \multirow{2}{*}{0.04} \\
\hline & Grupo experimental & 15.70 & 28 & & & \\
\hline \multirow{2}{*}{ Agresividad verbal } & Grupo control & 36.74 & 29 & \multirow{2}{*}{181.500} & \multirow{2}{*}{$0.000 *$} & \multirow{2}{*}{0.22} \\
\hline & Grupo experimental & 20.98 & 28 & & & \\
\hline \multirow{2}{*}{ Agresividad física } & Grupo control & 39.22 & 29 & \multirow{2}{*}{109.500} & \multirow{2}{*}{$0.000 *$} & \multirow{2}{*}{0.13} \\
\hline & Grupo experimental & 18.41 & 28 & & & \\
\hline \multirow{2}{*}{ Ira } & Grupo control & 40.69 & 29 & \multirow{2}{*}{67.000} & \multirow{2}{*}{$0.000 *$} & \multirow{2}{*}{0.08} \\
\hline & Grupo experimental & 16.89 & 28 & & & \\
\hline \multirow{2}{*}{ Hostilidad } & Grupo control & 41.40 & 29 & \multirow{2}{*}{46.500} & \multirow{2}{*}{$0.000 *$} & \multirow{2}{*}{0.06} \\
\hline & Grupo experimental & 16.16 & 28 & & & \\
\hline
\end{tabular}

Fuente: Elaboración propia

Nota: ${ }^{*} \mathrm{p}<0.05 ; \mathrm{n}=$ tamaño muestral; PSest= Probabilidad de Superioridad (tamaño del efecto).

El valor de la probabilidad de superioridad (tamaño del efecto) en el pretest de la variable agresividad total fue 0.48 , el cual se aproxima a un pequeño efecto y el valor en el postest fue 0.04, el cual se aproxima a no hay efecto en los estudiantes del grupo experimental sobre el grupo control. Por tanto, se concluye que el Programa "Fortaleciéndome" ha tenido efecto en el grupo experimental para disminuir la agresividad en los estudiantes del $5^{\circ}$ grado de primaria de la institución educativa pública 2033 Nuestra Señora de Fátima.

\section{Discusión}

El objetivo del presente trabajo fue determinar si la aplicación del programa "Fortaleciéndome" tiene una influencia significativa en la disminución de la agresividad de los estudiantes de primaria en una escuela de Lima Norte. En base a los resultados obtenidos $(Z=5,957>1,6706$ y el $\mathrm{p}<0,05$ ), es posible afirmar que "Fortaleciéndome" alcanzó el objetivo propuesto y redujo la agresividad física, verbal, como también la ira y la hostilidad. Resultados similares aunque en muestras y ámbitos geográficos distintos fueron conseguidos por Zenteno y Daza (2013) y su programa para la promoción de las habilidades sociales, y que logró una disminución de la agresividad hasta en un 99\% en una escuela de Lima Este. Bravo (2006) consiguió una reducción de agresividad física y verbal hasta en un 58,6\%, pero en niños venezolanos de educación inicial; para ello utilizó la técnica de economía de fichas en donde los juguetes actúan como reforzadores de conductas esperadas, y han sido seleccionados previamente por los niños durante la fase de la negociación.

Los programas de intervención contra la agresividad han surgido desde las escuelas, y por el interés y compromiso de los docentes. La escuela es un agente socializador fundamental, y los profesores son uno de los primeros referentes de los niños, junto a sus padres. Esto implica que las acciones contra las conductas agresivas solo pueden ser transversales para que alcancen sus resultados. Sin padres y profesores formados en habilidades comunicativas y sociales, sin padres, profesores y una comunidad comprometidos con una educación democrática y vigilante del consumo de violencia en los medios audiovisuales (López \& Ovejero, 2015) será muy difícil 
afrontar el problema cada vez más enraizado en la actual sociedad. Bravo (2006) recomendaba la capacitación y actualización del docente en torno a habilidades y destrezas para el fomento de relaciones afectivas en el aula, y del mismo modo para la organización de experiencias de aprendizaje que, realmente, promuevan una atmósfera de respeto, confianza y aceptación, con lo que se fortalece la autoestima del niño. Márquez (2017), en Ecuador, diseñó un plan de intervención cognitivo conductual para modificar conductas agresivas en niños y niñas de 7 años de edad, pero incluyendo al docente en el tratamiento estratégico. La estrategia parte de la premisa de que hay que conocer lo que el niño piensa de su entorno y de sí mismo, para dotarlo de una perspectiva sana y realista.

Si bien es cierto, la agresión es un producto de determinadas circunstancias socioeconómicas adversas (Dollar et al., 1939), eso no anula la posibilidad de que las situaciones sociales precarias puedan ser soportadas de distinta forma. Berkowitz (1969) sostuvo que la frustración personal es un intermediario fundamental al respecto, una especie de regulador lo que permitiría que algunos alumnos puedan verse afectados más que otros. La frustración, en tanto reacción emocional interna frente a la contrariedad, tiene un margen de excepción. Según Berkowitz (1993), muchos estudiantes pueden aprender a responder de forma no agresiva a las frustraciones, por lo que será decisivo el grado de equilibrio emocional del estudiante. En esta tarea, las instituciones educativas tienen un papel estelar, más aún si aprender a vivir juntos, el tercer pilar del informe Delors (1996), se experimenta -además del hogar- en la escuela. Por el tiempo que los estudiantes pasan en ella, es uno de los entornos de mayor importancia para su desarrollo social.

Sería un error creer que los niños desconocen el poder de la violencia. No son ángeles, pero son lo suficientemente humanos para rechazar las actitudes agresivas en su vida escolar, familiar y comunitaria. A este respecto, Quintana y Ramos (2013) encontraron que ese rechazo llegaba hasta un 58,33\% en una escuela de Lima Este, también en niños de $5^{\circ}$ grado de primaria. No cabe duda que el comportamiento agresivo infantil es un predictor confiable de conductas violentas en el joven y en el adulto, pero se puede hacer mucho frente a esta problemática, y a la vez contribuir a construir una sociedad mejor: desarrollar actividades que puedan contrarrestar el individualismo, la insensibilidad hacia los problemas ajenos y facilitar formas asertivas de comunicación y de autocontrol (Martínez, Tovar \& Ochoa, 2016). Esas formas asertivas vienen de la mano de las habilidades sociales. Un tratamiento pertinente para las conductas agresivas es el entrenamiento en la adquisición y desarrollo de habilidades sociales. A diferencia de décadas anteriores, esto ya no debe quedarse en lo declarativo, sino que es momento de pasar a la acción. No hay razón alguna para que permanezcan al margen de la escuela. Hay que incluirlas en el currículo, asignarles un horario y diseñar actividades didácticas (Caballo, 2007; Monjas y González, 1998).

Los niños en diferentes contextos observan cómo el mundo cambia cada vez más rápido y torna a la sociedad en algo mucho más complejo e imprevisible, ven lo influyentes que son los medios de comunicación y las redes sociales, no son ajenos a ciertas posibilidades de experimentar diversas opciones en lo afectivo, social, sexual, laboral y cognitivo (Muñoz, 2000). Asimismo, las referencias y modelos que podían proyectar los adultos se vuelven cada vez más cuestionables ("el que estudia, triunfa"), pareciera que el privilegio doblegó hace varias décadas al esfuerzo. No se los han contado, lo ven a diario con sus padres, porque éstos salen del hogar muy temprano y regresan a él muy tarde. Desde siempre escuchan que la familia es la célula básica de la sociedad, pero grupos fundamentalistas y ultraconservadores menosprecian a aquellas familias no clasificables en el modelo nuclear. Se les repite hasta el cansancio que "a la mujer se le respeta", "todos somos iguales", "di lo que piensas, pelea por lo que crees"; pero cuando la sociedad civil se moviliza y se organiza para hacerse escuchar ¿qué ocurre? Se les reprime ferozmente, se dispara perdigones a los ojos de los manifestantes, se introduce soda cáustica y gas pimienta en el agua lanzada por Carabineros, a las mujeres que protestan en las calles se les desprecia, se les ridiculiza, e inclusive se les amenaza con ultrajarlas sexualmente. Qué duda cabe, 
para formar a los niños los profesores y la escuela harán su parte. ¿Podrán hacerla los hogares y la sociedad?

\section{Referencias}

Álvarez, G. (2002). Introducción breve a la Psicología. Facultad de Psicología México D.F.: Universidad Nacional Autónoma de México.

Astor, R., Pitner, R. O., Benbenishty, R., \& Meyer, H. A. (2002). Public concern and focus on school violence. En L. A. Rapp-Paglicci, A. R. Roberts \& J. S. Wodarski (Eds.), Handbook of violence (pp. 262-302). New York: Wiley.

Atkinson, R. T., Atkinson, R. C., \& Hilgard, E. R. (1983). Pengantar Psikologi. Jakarta: Erlangga. Bandura, A. (1973). Aggression: A social learning analysis. Englewood cliffs, N.J.: Prentice Hall. Baron, R. \& Byrne, D. (2005). Psicología social (10ª ed.). Madrid: Pearson Educación, S.A.

Benítez, J. \& Justicia, F. (2006). El maltrato entre iguales: descripción y análisis del fenómeno. Electronic Journal of Research in Educational Psychology, 4(2), 151-170.

Berkowitz, L. (1969). The frustration-agression hypothesis revisited. In: L. Berkowitz (ed.), Roots of aggression (1-28). New York: Atherton Press.

Berkowitz, L. (1993). Aggression. New York: McGraw Hill.

Bravo, E. (2006). Efectos de un programa de modificación conductual para el manejo de conductas agresivas en niños de educación inicial (tesis de maestría). Centro de Investigaciones Psiquiátricas, Psicológicas y Sexológicas de Venezuela.

Brunstein, A., Kleinman, M., Altschuler, E., Marocco, F., Amakawa, L., \& Gould, M. S. (2011). High school bullying as a risk for later depression and suicidality. Suicide and LifeThreatening Behavior, 41(5), 501-516.

Buss, A. \& Perry, M. (1992). The Agression Questionnaire. Journal of Personality and Social Psychology, 63(3), 452-459.

Caballo, V. (2007). Manual de evaluación y entrenamiento de las habilidades sociales ( $\left.7^{\mathrm{a}} \mathrm{ed}.\right)$. Madrid: Siglo XXI Editores.

Castillo, N., Cruz, N., Peña, D., \& Cujiño, M. (2012). Descripción de los Factores de Riesgo y de Protección frente al Bullying de los estudiantes de bachillerato de un colegio de Acacías (Méta). Informe de tesis, Universidad de la Sabana, Colombia.

Castrillón, D. \& Vieco, F. (2002). Actitudes justificativas del comportamiento agresivo y violento en estudiantes universitarios de la ciudad de Medellín, Colombia. Revista Facultad Nacional de Salud Pública, 20(2): 51-66.

Cerezo, F. (2009). Bullying: análisis de la situación en las aulas españolas. International Journal of Psychology and Psychological Therapy, 9(3), 383-394.

Cerezo, F., \& Esteban, M. (1992). La dinámica bully-víctima entre escolares. Diversos enfoques metodológicos. Revista de Psicología Universitas Tarraconensis, 14(2), 131-145

Coccaro, E. (2003). Aggression: Psychiatric Assessment and Treatment. New York: Marcel Dekker.

Cohen, D., Nisbett, R., Bowdle, F. \& Schwarz, N. (1996). Insult, aggression, and the southern culture of honor: "an experimental ethnography". Journal of Personality and Social Psychology, 70(5), 945-960.

Currie, M. (2004). Doing anger differently: A group percussion therapy for angry adolescent boys. International Journal of Group Psychotherapy, 54 (3), 275-294.

Delors, J. (1996). La educación encierra un tesoro. Barcelona: Santillana, Unesco.

Díaz, Y. (2012). Bullying, acoso escolar elementos de identificación, perfil psicológico y consecuencias, en alumnos de educación básica y media. Psicología.com, 16(10), 24-37.

Dollard, J., Doob, L., Miller, N., Mowrer, O. \& Sears, R. (1939). Frustration and aggression. New Haven: Yale University Press.

Erceg-Hurn, D. \& Mirosevich, V. (2008). Modern Robust Statistical Methods An Easy way to Maximize the Accuracy and Power of Your Research. American Psychologist, 63(7), 591-601. 
Estévez, E., Jiménez, T., \& Musitu, G. (2008). Violence and victimization at school in adolescence. En D. H. Molina (Ed.), School psychology: 21st century issues and challenges, 79-115. New York: Nova Science Publishers.

Fadila, R. (2013). Hubungan Identitas Sosial dengan Perilaku Agresif pada Geng Motor. Psikologia: Jurnal Pemikiran dan Penelitian Psikologi, 8(2), 73-78.

Fernández, E. (1998). Psicología General. Motivación y Emoción. Madrid: Centro de Estudios Ramón Areces.

Fondo de las Naciones Unidas para la Infancia (2019). La Situación de las Niñas, Niños y Adolescentes en Lima. Lima: Fondo de las Naciones Unidas para la Infancia

Freire, P. (1997). Pedagogía de la autonomía. México: Siglo XXI Editores.

García, C. (2001). Investigación cuasiexperimental I: diseños preexperimentales y diseños cuasiexperimentales con grupos de control no equivalente. En: S. Fontes, C. GarcíaGallego, A.J. Garriga, M.C. Pérez-Llantada \& E. Sarría (Eds.), Diseños de investigación en Psicología, pp, 343-378. Madrid: Universidad Nacional de Educación a Distancia.

Gómez, A. (2005). Violencia e institución educativa. Revista Mexicana de Investigación Educativa, 10(26), 693-718.

Grissom, R.J. (1994). Probability of the superior outcome of one treatment over another. Journal of Applied Psychologist, 79(2), 314-316.

Instituto Nacional de Estadística e Informática (2015). Perú, Encuesta Nacional sobre Relaciones Sociales, 2015. Lima: Dirección Nacional de Censos y Encuestas.

Jenaabadi, H., \& Nastiezaie, N. (2011). A Comparison of aggression, anxiety and depression in high school students in the Saravan city. Journal of Urmia Nursing And Midwifery Faculty, 9(3), 12-25.

Kartono, M. (2005). ,Perbandingan Perilaku Agresif Antara Remaja yang Berasal dari Keluarga Bercerai dengan Keluarga Utuh '.Jurnal Psikologi, 3(1), 1-18.

Kirk, R. (1996). Practical Significance: A Concept Whose Time has Come. Educational and Psychological Measurement, 56(5), 746-759.

Ledesma, A. (1980). I Curso monográfico sobre agresividad. Madrid: Librería La Candela.

Little, T. D., Henrich, C. C., Jones, S. M., \& Hawley, P. H. (2003). Disentangling the "whys" from the "whats" of aggressive behaviour. International Journal of Behavioral Development, 27(2), 122-131.

López, L. \& Sabater, C. (2014). Medios audiovisuales y acoso escolar: buenas prácticas para la promoción de la convivencia. Revista de Investigación en Educación, 12(2), 23-30.

López, L. \& Ovejero, M. (2015). Habilidades comunicativas dentro de la familia: medida imprescindible contra el acoso escolar en La Rioja. Revista de Estudios de Educación (ESE). En prensa.

Lorenz, K. (1966). On aggression. New York: Harcourt, Brace, \& World.

Lorenz, K. (1974). Civilized Man's Eight Deadly Sins. New York: Hardcover.

Márquez, J. (2017). Plan de intervención cognitivo conductual para modificar conductas agresivas en niños y niñas de 7 años (tesis de grado). Universidad Técnica de Machala, Ecuador.

Martínez, W., Tovar, J., \& Ochoa, A. (2016). Comportamiento agresivo y prosocial de escolares residentes en entornos con altos niveles de pobreza. Revista Peruana de Medicina Experimental y Salud Publica, 33(3), 455-461.

Matalinares, M., Yaringaño, J., Uceda, J., Fernández, E., Huari, Y., Campos, A., \& Villavicencio, N. (2012). Estudio psicométrico de la versión española del Cuestionario de Agresión de Buss y Perry. Revista IIPSI, 15(1), 147-161. Facultad de Psicología de la Universidad Nacional Mayor de San Marcos.

Miguel, J., Casado, M., Cano, A. \& Spielberger, C. (1997). El estudio de la ira en los trastornos cardiovasculares mediante el empleo del Inventario de Expresión de Ira Estado-RasgoSTAXI. Ansiedad y Estrés, 3(1), 5-20.

Ministerio de Educación del Perú (2019). Sistema Especializado en reporte de casos sobre Violencia Escolar-SíseVe. Informe 2013-2018. 
Monjas, I. \& González, B. (1998). Las habilidades sociales en el currículo. Madrid: Ministerio de Educación, Cultura y Deporte; Secretaría General de Educación y Formación Profesional, España.

Mora, J. A., \& Ortega, R. (1997). El proyecto Sevilla anti-violencia Escolar: un modelo ecológico de intervención educativa contra el maltrato entre iguales. Conductas agresivas en la edad escolar. Madrid: Pirámide.

Muñoz, F. (2000). Adolescencia y agresividad (tesis doctoral). Universidad Complutense de Madrid, España.

Nansel, T. R., Overpeck, M., Pilla, R. S., Ruan, W. J., Simons, B., \& Scheidt, P. (2001). Bullying behaviors among US youth: Prevalence and association with psychosocial adjustment. Journal of the American Medical Association, 285(16), 2094-2100.

Novita, E. (2017). Perbedaan Agresivitas Ditinjau dari Pola Asuh Orangtua. Analitika, 4(2): 53 60.

Oliveros, M. \& Barrientos, A. (2007). Incidencia y factores de riesgo de la intimidación (bullying) en un colegio particular de Lima-Perú. Revista Peruana de Pediatría. 60(3), 150-155.

Organización de las Naciones Unidas (2017, 17 de enero). El acoso y la violencia escolar afecta a uno de cada cuatro niños. Washington, D.C.: Oficina Regional para las Américas de la Organización Mundial de la Salud

Organización Panamericana de la Salud (2002). Informe mundial sobre la violencia y la salud Washington, D.C.: Oficina Regional para las Américas de la Organización Mundial de la Salud.

Organización Panamericana de la Salud (2016). La prevención de la violencia juvenil: panorama general de la evidencia. Lima: Editorial Alpha

Pareja, J. A. (2002). La violencia escolar en contextos interculturales. Un estudio de la ciudad autónoma de Ceuta (Tesis doctoral). Universidad de Granada, España.

Pérez, V. (2011). Percepción de Gravedad, Empatía y Disposición a Intervenir en Situaciones de Bullying Físico, Verbal y Relacional en Profesores de $5^{\circ}$ a $8^{\circ}$ Básico. PSYKHE, 20(2), 25-37.

Piko, B., \& Keresztes, N. (2006). Physical activity, psychosocial health and life goals among youth. Journal of Community Health, 31(2), 136-145.

Plutchik, R. (1980). Emotion: A psychoevolutionary synthesis. New York: John Wiley.

Prieto, M., Carrillo, J., \& Jiménez, J. (2005). La violencia escolar: Un estudio en el nivel medio superior. Revista Mexicana de Investigación Educativa, 10(27), 1027-1045.

Quintana, C., \& Ramos, M. (2013). Actitudes hacia la agresividad de los estudiantes del quinto grado de educación primaria de la institución educativa Madre Admirable del distrito El Agustino, 2011 (tesis de maestría). Universidad César Vallejo, Lima, Perú.

Ramírez, J. M., \& Andreu, J. M. (2003). Aggression's typologies. International Review of Social Psychology, 16(3), 125-141.

Real Academia Española (2019). Diccionario de la lengua española. Lima: Edición del Tricentenario.

Rendón, M. (2008). Regulación de ira, tristeza y norma subjetiva en una muestra de escolares (Tesis de maestría). Universidad Nacional de Colombia, Bogotá, Colombia.

Román, M., \& Murillo, J. (2011). América Latina: violencia entre estudiantes y desempeño escolar. Revista CEPAL, 104.

Sáenz, M. (2010). Sintomatología depresiva y acoso escolar en un grupo de adolescentes escolares (Tesis de grado). Pontificia Universidad Católica del Perú, Lima, Perú.

Salgado, C (2012). Revisión de las investigaciones acerca del Bullying: Desafíos para su estudio. En L. Benites, J. Carozzo, V. Horna, L. Palomino, C. Salgado, C. Uribe \& L. Zapata, Bullying y Convivencia en la escuela. Aspectos conceptuales, aplicativos y de investigación. Lima: Asociación Latinoamericana para la Formación y Enseñanza de la Psicología.

Stein, A. H, Friedrich, L. K., \& Vondracek, F. (1972). Television content and young children's behavior. In J. P. Murray, E. A. Rubinstein, y G. A. Comstock (Eds.), Television and social behavior: Reports and papers (Vol. 2). Television and social learning, 202-317. Washington, DC: U.S: Government Printing Office. 
Tello, N. (2005). La socialización de la violencia en las escuelas secundarias. Proceso funcional a la descomposición social. Revista Mexicana de Investigación Educativa, 10 (27), 11651181.

Universidad Católica Sedes Sapientiae, UCSS (2010). Diagnóstico y Plan de Desarrollo Concertado 2011-2021. Lima: Municipalidad Distrital de Comas.

Viera, M., Fernández, I., \& Quevedo, G. (1989). Violence, bullying and counselling in the Iberica peninsula. En E. Roland \& E. Munthe (Eds.). Bullyind an international Perspective, 3552. London: David Fulton.

Zenteno, G. \& Daza, I. (2013). Efectos del programa de desarrollo de habilidades sociales para disminuir la agresividad de los alumnos de la institución educativa Fe y Alegría $N^{\circ} 26$ de la UGEL 05 de Lima, 2012 (tesis de maestría). Universidad César Vallejo, Lima, Perú.

Zimmer, M. J., \& Pronk, R. E. (2012). Relation of depression and anxiety to self- and peerreported relational aggression. Aggressive Behavior, 38(1), 16-30 